\title{
INDEFINITE GRAY-WHITE MATTER BORDER ON MRI AT TERM EQUIVALENT AGE IN PRETERM INFANTS WITH WHITE MATTER INJURY
}

\author{
B. van Kooij ${ }^{1}$, F. Groenendaal ${ }^{1}$, I. Isgum ${ }^{2}$, K. Kersbergen ${ }^{1}$, P. Anbeek ${ }^{1}$, L. de Vries ${ }^{1}$, M. Benders ${ }^{1}$ \\ ${ }^{I}$ Neonatology, Wilhelmina Children's Hospital / UMC, ${ }^{2}$ Image Sciences Institute, University Medical Center \\ Utrecht, Utrecht, The Netherlands
}

Background and aims: Both (regional) increase and decrease of white matter (WM) and cortical gray matter (cGM) volumes have been revealed in preterm infants compared to term born controls. The aim of this study was to analyse cGM and unmyelinated WM (UWM) volumes of preterm infants around term equivalent age with respect to WM injury (WMI).

Methods: MRI of the brain was performed in 81 preterm infants (gestational age: $28.5 \pm 1.6$ weeks, birth weight (BW) 1106 $\pm 308 \mathrm{~g} ; 39$ boys). Brain volumes were calculated using an in-house developed segmentation method (Anbeek PedRes 2008). Neonatal parameters and WMI (Woodward NEJM 2006) were related to cGM and UWM volumes.

Results: An unexpected increase in cGM volume/intracranial volume (ICV) ratio was seen in infants with lower BW Z-scores $\left(\mathrm{p}=0.001, \mathrm{R}^{2}=0.13\right)$. Additionally, we observed larger cGM volumes in preterm infants with more WMI $\left(\mathrm{R}^{2}=0.06, \mathrm{p}=0.03\right)$, and enlarged subarachnoid space $\left(\mathrm{R}^{2}=0.21, \mathrm{p}<0.001\right)$. In those infants, where the segmented cGM appeared thick, visual inspection revealed an indefinite cGM-UWM border. Infants with more WMI and larger subarachnoid space had lower UWM/ICV ratios.

Conclusions: An indefinite cGM-UWM border resulted in a relatively thick-segmented cGM in infants with more severe WMI and enlarged subarachnoid space. A decrease in UWM/ICV volume was associated with WMI. It may be suggested that an indefinite cGM-UWM border, causing an erroneously larger cGM volume, was due to an adverse effect on proliferation and organisation of neuroblasts in infants with WMI. Further studies will be needed to quantify this observation. 